\title{
Non-Hodgkin's lymphoma: unexpected cause of shoulder pain. A systematic review of the literature
}

\author{
Manlio Fabio Caporale ${ }^{1}$ \\ Giovanni Francesco Gambino ${ }^{1}$ \\ Fabio Saverio Larosa ${ }^{1}$ \\ Angelo Del Buono ${ }^{2}$ \\ Federico Di Segni ${ }^{1}$ \\ 1 Department of Orthopaedic and Trauma Surgery, \\ Hospital SS Gonfalone, Monterotondo (RM), Italy \\ 2 Department of Orthopaedic and Trauma Surgery, \\ Hospital Antonio Cardarelli, Campobasso, Italy
}

Corresponding author:

Angelo Del Buono

Department of Orthopaedic and Trauma Surgery

Hospital Antonio Cardarelli,

86100 Contrada Tappino

Campobasso, Italy

\section{Summary}

The non-Hodgkin lymphoma (NHL) is one of the most common shoulder neoplasms, especially the diffuse large $B$ cell lymphoma (DLBCL). We report a rare case of shoulder pain in a 80 -yearold man presenting with a six-month history of continuous severe pain to the right shoulder. Routine laboratory studies were normal. Shoulder MRI showed an in-growing inhomogeneous lesion in the anteromedial aspect of the right humeral head extended within the cortical bone of the humerus (osteolitic lesion), next to the surrounding soft tissues. He also underwent shoulder arthroscopy: the intra-articular involvement of the shoulder was therefore excluded. A percutaneous bone biopsy performed on the same day was diagnostic for lymphoma. Three days later, the patient underwent surgical excision of the mass; a reverse shoulder prosthesis was then implanted (Aequalis reversed prosthesis). The patient started chemiotherapy according with CHOP (cyclophosphamide, doxorubicin, vincristine, and prednisone) regimen, but did not tolerate it because of the sudden onset of herpes zoster. At 9month follow-up, the patient is doing well, with fair range of motion, due to the delay of rehabilitation, but no shoulder pain and no evidence of local or systemic recurrence. A painful shoulder may be due to lymphoma even in the absence of classical symptoms. In suspected patients, plain radiographs should be followed by magnetic resonance imaging and bone biopsy. Tumor removal and shoulder arthroplasty can be an effective therapy. Given the devastating side effects of adjuvant chemotherapy, we do not recommend it in elderly patients.

KEY WORDS: shoulder pain; non-Hodgkin lymphoma; surgery; differential diagnosis.

\section{Introduction}

Shoulder pain is often controversial: rheumatoid arthritis (RA), osteoarthritis, osteonecrosis, rotator cuff arthropathy, traumatic arthritis, fractures, failed surgery, cervical spine disorders, and neoplasms may cause it ${ }^{1}$. Even though most shoulder affections are diagnosed by history, physical examination, and radiographic imaging, diffuse neoplasms of this area may be better assessed at histopathology². Among these latter, the non-Hodgkin lymphoma (NHL) is one of the most common, especially the diffuse large B cell lymphoma (DLBCL). The incidence of $\mathrm{NHL}$ is increasing, accounting for 40,000 cases per year in the United States $^{3}$, mostly in patients with acquired immunodeficiency or taking immunosuppressive drugs. Often lethal, it usually occurs with multiple lymphadenopathies, but any site containing lymphoid tissue, included the bone marrow, may be involved. The involvement of soft tissues is primary in less than $1 \%{ }^{4}$ and, when present, is usually secondary to a direct spreading from affected surrounding lymphoidal sites and/or metastatic hematogenous dissemination ${ }^{5}$. From a clinical point of view, lymphomas usually manifest with a soft tissue mass, swelling, and pain. Most patients are asymptomatic whereas the main presentation is a painless adenopathy in the neck or supraclavicular region $^{6}$. Biopsy with pathological examination is diriment in almost all instances. We report a rare case of shoulder pain in a patient affected by non-Hodgkin lymphoma (NHL) of the right shoulder. We have also performed a review on the shoulder lymphoma.

\section{Case presentation}

A 80-year-old man presented with a six-month history of continuous severe pain to the right shoulder. He was taken to our department of orthopedic and trauma surgery in October 2012. He denied any constitutional symptoms, including fever, weight loss, or night sweats. The general physical examination was unremarkable for a neoplastic disease, with no adenopathies or hepatosplenomegaly. Physical examination was remark- 
able for limited range of motion and pain in all planes of movement. The active range of motion of the right shoulder was $20^{\circ}$ abduction, $20^{\circ}$ external rotation, $35^{\circ}$ flexion, and $50^{\circ}$ internal rotation. Plain radiographs of the shoulder showed just mild gleno-humeral arthritis and their report was negative (Fig. 1). Routine laboratory studies were normal. The cervical spine MRI showed extensive signs of spondyloarthritis, compatibly with the patient age. Shoulder MRI showed an in-growing inhomogeneous lesion in the anteromedial aspect of the right humeral head extended within the cortical bone of the humerus (osteolitic lesion), next to the surrounding soft tissues. The TSE sequence with deletion of the T2 signal from adipose tissue showed a marked inhomogenous hyperintense signal (Fig. 2). The margins appeared unclean and irregular, with evidence of infiltration, osteolysis of the cortical bone, bone marrow involvement, extended to surrounding soft tissue, nearby the subscapularis muscle. At the end of October, he underwent shoulder arthroscopy: the intra-articular involvement of the shoulder was therefore excluded. A percutaneous bone biopsy performed on the same day was diagnostic for lymphoma. Three days later, the patient underwent surgical excision of the mass. The procedure was made under general anesthesia through the anterior, delto-pectoral approach to the shoulder. A transverse fracture line lay from the head to the upper third of the diaphysis. The proximal third of the humerus was removed, up to the distal insertion of the deltoid muscle, and sent for histology. A reverse shoul-
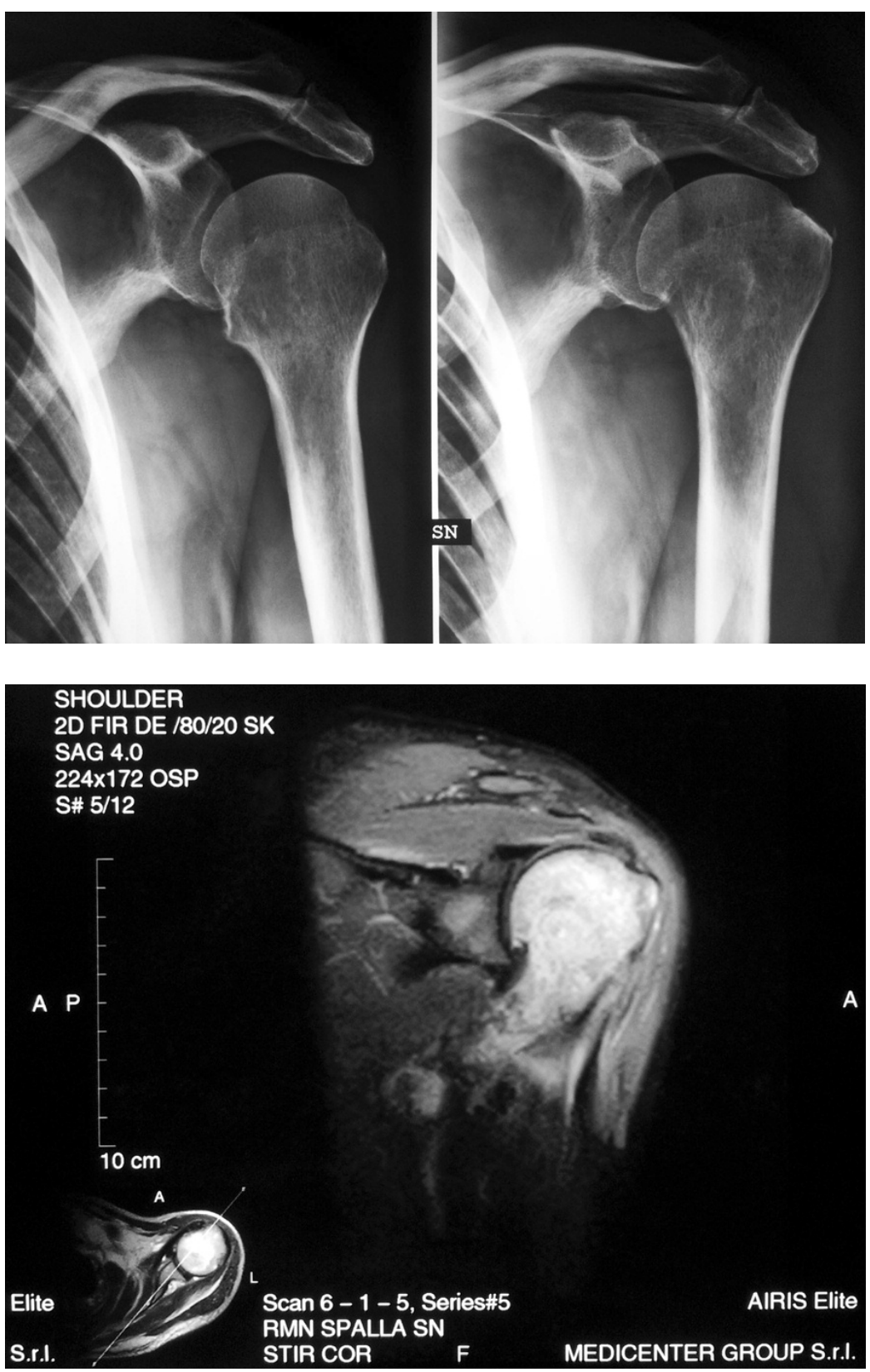

Figure 1. X-rays examination of the left proximal humerus, showing just a little medial erosion; radiology report pointed out only acromio-clavicular arthritis and sclerosis of the greater tuberosity.
Figure 2. MRI examination of the left shoulder, showing an impressive proximal humeral hyperintensity with extension to the subscapularis tendon. 
der prosthesis was then implanted (Aequalis reversed prosthesis) (Fig. 3). Histology showed a diffuse proliferation of large lymphoid cells with irregular round or oval nuclei (pleomorphic) or multinucleated, marked granules, and wide cytoplasm, mixed with small CD3+ Tlymphocytes. Diffuse necrosis was also present. Immune-histochemistry of paraffin sections showed different immune-phenotypes. Neoplastic cells were CD3, CD5, CD30, S100, CKMNF116 negative, CD20, LCA positive with 55 to $60 \%$ Ki67 positive nuclei. A final diagnosis of diffuse large cells B-non Hodgkin lymphoma was made. The patient also started chemiotherapy ac-

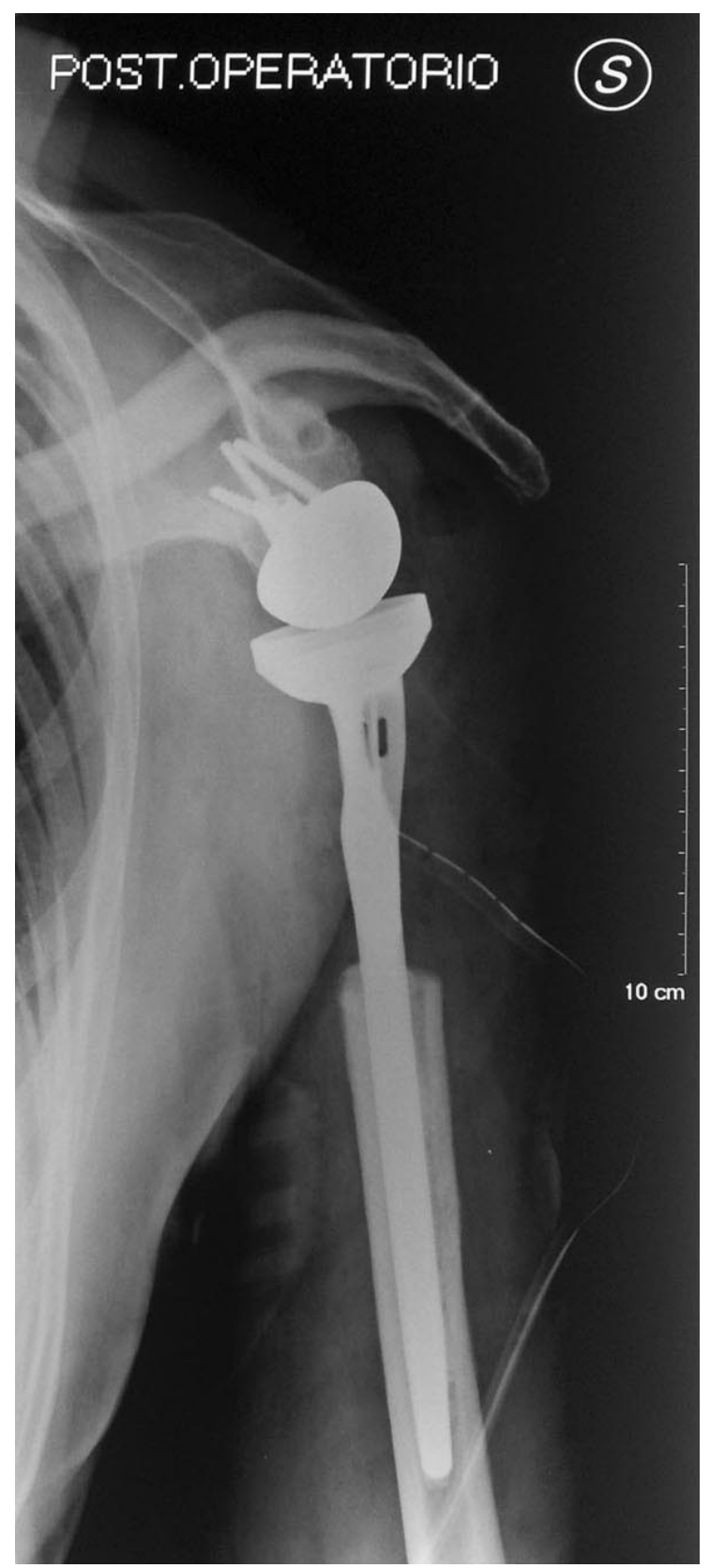

Figure 3. Post-operative X-ray examination after en bloc resection of the tumour and shoulder replacement with reverse prosthesis. cording with CHOP (cyclophosphamide, doxorubicin, vincristine, and prednisone) regimen, but did not tolerate it because of the sudden onset of herpes zoster. At 9-month follow-up, the patient was doing well, with fair range of motion, due to the delay of rehabilitation, but no shoulder pain and no evidence of local or systemic recurrence.

\section{Search of the literature}

We performed a systematic search of the literature using the combined keywords "non-Hodgkin lymphoma" "shoulder pain" "shoulder imaging" "diagnosis" with no limit for year of publication and level of evidence of the study. Medline (PubMED), EMBASE, CINHAL, Cochrane, Sports Discuss and Google scholar databases were accessed up to 20 September 2013 to identify articles published in English, Spanish, French, Portuguese and Italian in peer-reviewed journals, reporting data and information on lymphoma presentation to the shoulder. Studies on animals, cadavers, in vitro or animal studies, letters to editors, and editorials were also excluded. Two authors (ADB and FDS) independently assessed the abstract of each publication, selecting on the basis of its content, and excluding articles without abstract available. When inclusion or exclusion was not possible based on the abstract, the full-text versions were downloaded. The reference lists of the selected articles were reviewed by hand to identify articles not included at the first electronic search.

\section{Search Results}

We identified 5 publications relevant to the topic, $4^{7}, 3,8,9$ case reports and 1 retrospective study ${ }^{10}$, on patients with shoulder girdle neoplasms, published from 1999 to 2013. The total number of patients was 5 ( 2 males, 3 females); the average age at time of diagnosis was 49.5 years. Concomitant immunological morbidities were present in 2 patients: HIV in 1 patient, rheumatoid arthritis in 1. Diagnosis was undertaken by clinical examination, MRI, scintigraphy, and pathological investigations including histology and immuno-histochemistry. All patients underwent chemotherapy. Only one patient underwent shoulder joint arthroplasty. Information on the last follow up were reported in only 2 studies, 7 months $^{3}$ and 4 years $^{3}$, respectively. Both patients were free of symptoms with no evidence of local or systemic pathology.

\section{Discussion}

This is the first case of lymphoma of the shoulder occurring with isolated pain and limited range of motion of the right shoulder in a patient with normal general examination without any associated immunological disorder. Another case of shoulder lymphoma has been found in a patient who had undergone shoulder arthroplasty after prolonged immunosuppressive therapy tak- 
en because affected by long-standing rheumatoid arthritis $^{3}$. Lymphoma may also be associated with lupus erythematosus ${ }^{11}$, scleroderma, and Sjögren's syndrome. In addition, prolonged immunosuppressive therapies may increase the risk for neoplasms of the immune system ${ }^{12}$. Methotrexate has been implicated in some reversible lymphomas by decreasing the host's immune response, and the development of these tumors may be associated with the functional level of immune suppression ${ }^{3}$. Primary bone $\mathrm{NHL}$ in the absence of extra skeletal disease has also been reported in HIV patients, involves mainly the lower extremities, spine, pelvis and skull and presents with fever, painful unilateral limb swelling, weight loss and pathologic fracture ${ }^{9}$. The presentation of this case is unusual. Pain was firstly attributed to cervical spine spondylosis and rotator cuff arthropathy. A shoulder lymphoma has to be differentiated from neoplasms involving the soft tissue, mostly the sarcoma, osteomielitis, spine affections, and neurological disorders. Radiographs and MRI showed gleno-humeral arthritis and rotator cuff tendinopathy, but that evidence was too mild to justify the intensity of pain. In non-Hodgkin's lymphomas, even though radiographic assessment may be negative, MRI is more specific to assess the extent of the mass within the bone and surrounding soft tissues. Bone marrow changes are seen as areas of hypo-intensity on T1-weighted images and as areas of hyper-intensity on STIR images or fat-saturated fast spin echo T2-weighted images. The associated soft-tissue mass appears hyper-intense on T2weighted images. CT and scintigraphy have a complementary role. Imaging findings are similar to those in osteomyelitis, and clinical correlation is often needed. Clinically, bone pain is present only if the bone marrow is affected. Biopsy is needed to diagnose the marrow involvement and definitively stage it. We first performed a shoulder arthroscopy, not to repair rotator cuff but to exclude any intra-articular involvement, and soon after a percutaneous bone biopsy. Three days later, the patient underwent shoulder arthroplasty. We used the delto-pectoral approach to obtain an abundant resection of surrounding soft tissues as lymphoma may present with extensive infiltration of the perinodal adipose tissue ${ }^{13}$. Given the large dimension of the mass, we performed a large excision of both bone and soft tissues with tumor-free margins. We acknowledge that the first management of this condition is conservative, and patients are well responsive to chemotherapy with or without radiotherapy but, in our patient, the extent of the mass was excessive. We implanted at the same stage a reverse prosthesis of the shoulder to restore congruity and biomechanics of the shoulder, otherwise impaired by the extended resection of the infiltrated subscapularis tendon. After surgery, the patient started chemotherapy, but he stopped the cycle after 2 sessions as he reported side effects. The effects of chemotherapy were deleterious. Specifically, the patient developed a iatrogenic form of herpes zoster and secondary post-herpetic neuralgia, slowing down the rehabilitation protocol. This report does not allow to draw any conclusion about the best treatment and long term outcomes of these patients. Larger studies with long-term follow-ups are needed to better define the evolution of this pathology.

\section{Conclusion}

A painful shoulder may be due to lymphoma even in the absence of classical symptoms. In suspected patients, plain radiographs should be followed by magnetic resonance imaging and bone biopsy. Tumor removal and shoulder arthroplasty can be an effective therapy. Given the devastating side effects of adjuvant chemotherapy, we do not recommend it in elderly patients.

\section{References}

1. Del Buono A, Oliva F, Osti L, Maffulli N. Metalloproteases and tendinopathy. Muscles, Ligaments and Tendons Journal 2013 May 21; 3(1):51-7.

2. Wirth $\mathrm{M}$, Rockwood C. Complications of shoulder arthroplasty. Clin Orthop Relat Res 1994; 307:47-69.

3. Arredondo J, Worland R, Sinnenberg R, Qureshi G. NonHodgkin's lymphoma as an unexpected diagnosis in a shoulder arthroplasty. J Arthroplasty 1999; 14:108-111.

4. Yang J, Zhang F, Fang H, Ye Z, Lin S, Han A. Clinicopathologic features of primary lymphoma in soft tissue. Leuk Lymphoma 2010; 51:2039-2046.

5. Lanham G, Weiss S, Enzinger F. Malignant lymphoma. A study of 75 cases presenting in soft tissue. Am J Surg Pathol 1989; 13:1-10.

6. Liu Y, Chang W, Gau J, Chao T. Primary bone lymphoma of the shoulder. Br J Haematol 2012; 158:677.

7. Li Z, Li P, Wang Z, Huang G. Primary extranodal soft-tissue B-cell lymphoma with abundant immunoglobulin inclusions mimicking adult rhabdomyoma: a case report. J Med Case Rep 2011; 5:53.

8. Robinson D, Halperin N, Agar G, Alk D, Rami K. Shoulder girdle neoplasms mimicking frozen shoulder syndrome. J Shoulder Elbow Surg 2003; 12:451-455.

9. Marotta D, Sgambato A, Cerciello S, Magarelli N, Martini M, Larocca L, Maccauro G. Soft tissue non-Hodgkin lymphoma of shoulder in a HIV patient: a report of a case and review of the literature. World J Surg Oncol 2008; 6:111.

10. Cleeman E, Auerbach J, Springfield D. Tumors of the shoulder girdle: a review of 194 cases. J Shoulder Elbow Surg 2005; 14:460-465.

11. Abu-Shakra M, Gladman D, Urowitz M. Malignancy in systemic lupus erythematosus. Arthritis Rheum 1996; 39:10501054.

12. Newkirk M, Shiroky J, Johnson N, Danoff D, Isenberg D, Shustik C, Pearson G. Rheumatic disease patients, prone to Sjögren's syndrome and/or lymphoma, mount an antibody response to BHRF1, the Epstein-Barr viral homologue of $\mathrm{BCL}$ 2. Br J Rheumatol 1996; 35: 1075-1081.

13. Weiss LM CW, Schnitzer B. Lymph nodes, p. 1115. In Damjanov I, Linder J (eds): Anderson's pathology. Mosby-Year Book, St Louis, 1996. 